\title{
Qualidade fisiológica de sementes de diferentes árvores matrizes de Poincianella pyramidalis (Tul.) L. P. Queiroz ${ }^{1}$
}

\author{
Physiological quality of seeds from different parent trees of Poincianella pyramidalis \\ (Tul.) L. P. Queiroz
}

\author{
Cosmo Rufino de Lima ${ }^{2}$, Riselane de Lucena Alcântara Bruno ${ }^{3}$, Katiane da Rosa Gomes da Silva**, Mauro \\ Vasconcelos Pacheco ${ }^{5}$ e Edna Ursulino Alves ${ }^{3}$
}

\begin{abstract}
RESUMO - A catingueira [Poincianella pyramidalis (Tul.) L. P. Queiroz] é uma espécie arbórea endêmica do bioma Caatinga, bastante explorada devido ao seu potencial madeireiro, forrageiro e farmacológico. Face à relevância de sua importância, este trabalho teve o objetivo de avaliar a qualidade fisiológica das sementes entre matrizes de P. pyramidalis por meio de testes fisiológicos para fins de restauração ambiental. O estudo de campo foi conduzido na Fazenda Açude, município de Soledade-PB, onde foram selecionados 28 matrizes de catingueira, sendo as análises, realizadas no Laboratório de Análise de Sementes do Centro de Ciências Agrárias da Universidade Federal da Paraíba. Foram avaliadas as seguintes variáveis: teor de água, peso de mil sementes, número de sementes por quilograma, germinação, vigor \{primeira contagem e índice de velocidade de germinação, comprimento e massa seca de plântulas, emergência, índice de velocidade e primeira contagem de emergência, envelhecimento acelerado (procedimento tradicional e solução saturada de $\mathrm{NaCl}$ ) \}. O delineamento experimental utilizado foi inteiramente casualizado, com os dados submetidos à análise de variância e a comparação entre as médias realizadas pelo teste de Scott-Knott a $5 \%$ de probabilidade. Com base nos resultados, a qualidade fisiológica das sementes de Poincianella pyramidalis varia mesmo quando oriundas de matrizes de uma única área de coleta; o teste de envelhecimento acelerado, utilizado no método tradicional, no período de 24 horas e à temperatura de $41{ }^{\circ} \mathrm{C}$ mostra ser o teste de vigor mais eficiente na avaliação do potencial fisiológico das sementes de $P$. pyramidalis.
\end{abstract}

Palavras-chave: Vigor. Caatinga. Semiárido. Catingueira.

\begin{abstract}
Known locally as 'catingueira', Poincianella pyramidalis (Tul.) L. P. Queiroz is an arboreal species endemic to the Caatinga which is extensively exploited for its timber, forage and pharmacological potential. Given the relevance of this importance, the objective of this work was to evaluate the physiological quality of seeds among parent trees of P. pyramidalis using physiological tests, for the purposes of environmental restoration. The field study was carried out at the Fazenda Açude Farm, in the town of Soledad, Paraíba, Brazil, where 28 parent catingueira trees were selected. The analyses were done at the Laboratory for Seed Analysis, in the Centre for Agricultural Sciences of the Federal University of Paraíba. The following variables were evaluated: water content, thousand seed weight, number of seeds per kilogram, germination, vigor \{first count and rate of germination index, seedling length and dry weight, emergence, emergence rate index and emergence first count, accelerated aging (traditional procedure and saturated $\mathrm{NaCl}$ solution) \}. The experimental design was completely randomized, with the data undergoing variance analysis and the comparison of averages using the Scott-Knott test at 5\% probability. Based on the results, the physiological quality of Poincianella pyramidalis seeds varies even when coming from trees in the same area, the accelerated aging test as used in the traditional method, for a period of 24 hours and at a temperature of $41^{\circ} \mathrm{C}$, is shown to be the most efficient means of testing vigour when evaluating the physiological potential of the seeds of P. Pyramidalis.
\end{abstract}

Key words: Vigour. Caatinga. Semiarid. Catingueira.

\footnotetext{
* Autor para correspondência

'Recebido para publicação em 29/05/2013, aprovado em 26/12/2013

Parte da Tese do primeiro autor, pesquisa financiada pelo CNPq

2Programa de Pós-Graduação em Agronomia/CCA-UFPB, Areia-PB, Brasil, cosmoagr@hotmail.com

${ }^{3}$ Departamento de Fitotecnia e Ciências Ambientais/CCA-UFPB, Campus II, Areia-PB, Brasil, lanebruno.bruno@gmail.com; ednaursulino@cca.ufpb.br ${ }^{4}$ Programa Nacional de Pós-Doutorado (PNPD), Universidade Federal da Paraíba, Areia-PB, Brasil, katrgs@gmail.com

${ }^{5}$ Universidade Federal do Rio Grande do Norte (UFRN), Unidade Acadêmica Especializada em Ciências Agrárias (UECIA), Macaíba-RN, pachecomv@hotmail.com
} 


\section{INTRODUÇÃO}

O semiárido do nordeste brasileiro apresenta uma grande diversidade de espécies, que compõem o estrato herbáceo, arbustivo e arbóreo. Dentre as espécies que ainda carecem de estudos encontra-se a catingueira [Poincianella pyramidalis (Tul.) L. P. Queiroz], endêmica do Bioma Caatinga com múltiplas utilidades tais como: potencial madeireiro, medicinal, uso veterinário, restauração florestal, forragem para o gado e aplicações industriais (MAIA, 2004).

Nesse sentido, pesquisas que busquem estudar o potencial das espécies endêmicas da Caatinga são de grande importância, principalmente quando surgem políticas públicas a exemplo da Lei $\mathrm{n}^{\circ} 10.711$, de 5 de agosto de 2003; de acordo com o capítulo XII, da Lei de sementes e mudas, o qual faz abordagem acerca "das espécies florestais, nativas ou exóticas e das de interesse medicinal ou ambiental", a coleta de sementes florestais nativas é normalmente realizada para a recomposição de áreas de preservação permanentes, produção de madeira ou outros produtos não madeireiros (DAVIDE; SILVA, 2008).

Para obtenção de sementes de boa qualidade devem-se escolher plantas que apresentem características superiores às demais da mesma espécie, efetuando-se os procedimentos de marcação e mapeamento no campo, monitoramento, coleta e qualidade das sementes para que futuramente se possa manipular o material corretamente (HIGA; SILVA, 2006).

Após as operações de coleta, extração e beneficiamento segue-se a etapa do controle de qualidade das sementes em laboratório, cujo objetivo principal é determinar o valor das sementes de uma matriz antes de ser selecionada a árvore como produtora de sementes. Dentre as análises usuais, a determinação do teor de água é essencial, além da avaliação da qualidade física realizada por meio dos testes de peso de mil sementes, número de sementes por quilograma e caracterização biométrica.

Para se determinar o nível de qualidade das sementes, um dos meios utilizados é o teste de germinação, realizado sob condições de temperatura e substratos ideais para cada espécie (PASSOS et al., 2008). Entretanto, este teste pode ter pouca eficiência para estimar o desempenho no campo, onde as condições nem sempre são favoráveis. Desta forma, os resultados de emergência das plântulas em campo podem ser consideravelmente inferiores ao teste de germinação em laboratório (GUEDES et al., 2009).

A tecnologia de sementes, como segmento do processo de produção, tem procurado aprimorar os testes usados para avaliar o potencial fisiológico das mesmas, com o objetivo de que os resultados expressem o máximo de desempenho dos lotes de sementes sob condições de campo (DUTRA; VIEIRA 2004).

Diante dessas constatações, foram desenvolvidos vários métodos para avaliar o vigor de sementes, como complemento ao teste de germinação (GARCIA; NOGUEIRA; ABREU, 2004). Dentre eles o teste de envelhecimento acelerado tem sido bastante utilizado (TORRES; NEGREIROS, 2008). Esse teste tem como princípio promover uma rápida deterioração de sementes, quando expostas a condições adversas de temperatura e umidade relativa do ar, os quais são os fatores ambientais mais relacionados à deterioração (GARCIA; NOGUEIRA; ABREU, 2004).

Diante do exposto e da importância da espécie, o presente trabalho teve por objetivo avaliar a qualidade fisiológica das sementes de $P$. pyramidalis obtidas de diferentes árvores matrizes.

\section{MATERIAL E MÉTODOS}

O estudo de campo foi conduzido na Fazenda Açude, município de Soledade-PB, na microrregião do Curimataú Ocidental, no Agreste Paraibano, entre as coordenadas $-7^{\circ} 7^{\prime} 22^{\prime \prime}$ de latitude e $-36^{\circ} 19^{\prime} 33^{\prime \prime}$ de longitude, localizada a $10 \mathrm{~km}$ da rodovia BR-230.

As 28 matrizes selecionadas estavam distribuídas por toda a área, as quais possuíam grande número de inflorescências, boa formação da copa e aparência fitossanitária, bem como com altura variando entre 1,5 a 4 metros; em seguida, estas foram georeferenciadas e catalogadas em fichas de identificação individual.

Os frutos foram colhidos manualmente, tomandose o cuidado para não provocar danos mecânicos nos mesmos e nas sementes. Imediatamente após a colheita, as amostras de frutos e sementes foram encaminhadas ao Laboratório de Análise de Sementes do Centro de Ciências Agrárias da Universidade Federal da Paraíba (LAS/CCA/ UFPB), para a realização das seguintes avaliações (físicas e fisiológicas):

Teor de água das sementes: determinado pelo método da estufa a $105 \pm 3{ }^{\circ} \mathrm{C}$ por 24 horas de acordo com Brasil (2009), com quatro repetições de 25 sementes cada;

Peso de mil sementes: foi efetuada através da pesagem de oito subamostras de 100 sementes, em balança analítica com sensibilidade de 0,001 g. O peso de 100 sementes foi calculado pela multiplicação do peso médio obtido nas subamostras por 10, de acordo com as recomendações das Regras para Análise de Sementes (BRASIL, 2009); 
Número de sementes por quilograma: calculouse o número de sementes por quilograma em oito repetições de 100 sementes de cada matriz, de acordo com metodologia descrita por Oliveira (2007);

Teste de germinação: as sementes foram desinfestadas em solução de hipoclorito de sódio (5\% i.a. durante 1 minuto) sendo, em seguida, submetidas ao teste de germinação em germinador tipo Biochemical Oxigen Demand (B.O.D.), regulada a regime alternado de temperatura $20-30{ }^{\circ} \mathrm{C}$ (LIMA et al., 2011) e fotoperíodo de oito horas, onde a temperatura mais alta correspondeu ao período luminoso simulado através de lâmpadas fluorescentes tipo luz do dia $(4 \times 20 \mathrm{~W})$. As sementes foram distribuídas entre o substrato areia, previamente esterilizado em autoclave a $120^{\circ} \mathrm{C}$ durante $2 \mathrm{~h}$, dispostas em caixas acrílicas transparentes (gerbox) com dimensões de 11 x 11 x $3,5 \mathrm{~cm}$. O substrato foi umedecido com água destilada a $60 \%$ de sua capacidade de retenção de água, segundo Brasil (2009). O número total de sementes germinadas ao $13^{\circ}$ dia após a semeadura foi avaliado o (BRASIL, 2013; LIMA et al., 2011), sendo os resultados expressos em porcentagem de plântulas normais;

Primeira contagem de germinação: correspondeu à porcentagem acumulada de plântulas, observandose a emergência dos cotilédones e o surgimento do hipocótilo, ao $6^{\circ}$ dia após o início do teste de germinação (BRASIL, 2013; LIMA et al., 2011);

Índice de velocidade de germinação (IVG): foi determinado de acordo com a equação proposta por Maguire (1962);

Comprimento de plântulas: ao final do teste de germinação, o comprimento de plântulas normais de cada repetição foi determinado com o auxílio de uma régua graduada em centímetros, sendo os resultados expressos em centímetros por plântula;

Massa de matéria seca de plântulas: foi determinada acondicionando as plântulas normais de cada repetição em estufa com circulação de ar forçada, regulada a $65{ }^{\circ} \mathrm{C}$, até atingirem peso constante. A pesagem do material seco foi realizada em balança com precisão de 0,001 g e os resultados expressos em gramas por plântula (NAKAGAWA, 1999);

Emergência em campo: a avaliação da emergência foi realizada em canteiros localizados na área experimental do CCA/UFPB, com semeadura a $2 \mathrm{~cm}$ de profundidade, após desinfestação das sementes em solução de hipoclorito de sódio $5 \%$ i.a. durante 1 minuto. Foram realizadas contagens diárias do número de plântulas normais, observando-se a emergência dos cotilédones e hipocótilo, até a estabilização da emergência. Os resultados foram expressos em porcentagem de plântulas normais;
Primeira contagem de emergência: correspondeu à porcentagem acumulada de plântulas normais ao $6^{\circ}$ dia após o início do teste;

Índice de velocidade de emergência (IVE): determinado de acordo com a equação proposta por Maguire (1962);

Envelhecimento acelerado: foram utilizadas sementes provenientes das matrizes classificadas como superiores pelo teste de germinação, em quantidade suficiente para distribuição uniforme das mesmas sobre uma tela de alumínio fixada no interior de caixas plásticas do tipo gerbox, funcionando como compartimento individual (mini-câmara). No interior dessas minicâmaras foram adicionados $40 \mathrm{~mL}$ de água destilada e, em seguida, as mesmas foram transferidas para um germinador tipo B.O.D. regulado a $41{ }^{\circ} \mathrm{C}$, durante três períodos de envelhecimento (24; 48 e 72 horas). Após esse período, quatro repetições de 25 sementes por tratamento foram submetidas ao teste de germinação, computandose a porcentagem de plântulas normais por repetição. O teor de água das sementes, antes e após os diferentes períodos de envelhecimento, foi determinado através do método da estufa a $105 \pm 3{ }^{\circ} \mathrm{C}$ por $24 \mathrm{~h}$, utilizando-se duas subamostras com 20 sementes cada (BRASIL, 2009);

Envelhecimento acelerado com solução saturada de $\mathrm{NaCl}$ : conduzido de forma semelhante ao descrito para o procedimento tradicional, adicionandose, porém, ao fundo de cada caixa plástica, $40 \mathrm{~mL}$ de solução saturada de $\mathrm{NaCl}$ (40 g de sal/100 mL de água destilada), estabelecendo um ambiente com $76 \%$ de UR (JIANHUA; MCDONALD, 1996).

\section{RESULTADOS E DISCUSSÃO}

$\mathrm{Na}$ análise de variância para as variáveis peso de mil sementes e número de sementes por quilograma (Tabela 1) constatou-se efeito significativo a $5 \%$ de probabilidade pelo teste $\mathrm{F}$.

O teor inicial de água das sementes provenientes de cada planta de catingueira não apresentou grande variação (menor ou igual a 2\%) (Tabela 2). Esse fato é importante na execução dos testes de vigor, considerando que a uniformização do teor de água das sementes é imprescindível para a padronização das avaliações e obtenção de resultados consistentes (MARCOS FILHO, 1999).

Em relação ao peso de mil sementes e número de sementes por quilograma (Tabela 2), houve variação de 97,29 a 196,01 g e 5.103 a 10.293 sementes $\mathrm{kg}^{-1}$, respectivamente. Estes dados estão de acordo com a Instrução Normativa de 17 de janeiro de 2013, que 
Tabela 1 - Resumo da análise de variância de sementes de vinte oito matrizes de $P$. pyramidalis, provenientes de Soledade-PB

\begin{tabular}{lccc}
\hline \multirow{2}{*}{ Fonte de variação } & Grau de liberdade & \multicolumn{2}{c}{ Quadrados médios } \\
\cline { 3 - 4 } & & Peso de Mil Sementes & N $^{\circ}$ de Sementes por Quilograma \\
\hline Matrizes & 27 & $3184,13^{*}$ & $9000951,75^{*}$ \\
Resíduo & 84 & 10,66 & 33695,23 \\
CV $(\%)$ & & 2,24 & 2,52 \\
\hline
\end{tabular}

*significativo a $5 \%$ pelo teste $\mathrm{F}$

Tabela 2 - Teor de água, peso de mil sementes e número de sementes por quilograma de vinte e oito matrizes de $P$. pyramidalis, provenientes de Soledade-PB

\begin{tabular}{cccc}
\hline Matrizes & Teor de Água das Sementes $(\%)$ & Peso de Mil Sementes $(\mathrm{g})$ & $\mathrm{N}^{\mathrm{o}}$ de Sementes por Quilograma \\
\hline 14 & 11,6 & $196,01 \mathrm{a}$ & $5103 \mathrm{n}$ \\
22 & 12,5 & $193,68 \mathrm{a}$ & $5165 \mathrm{n}$ \\
29 & 13,1 & $182,44 \mathrm{~b}$ & $5482 \mathrm{~m}$ \\
15 & 11,9 & $174,34 \mathrm{c}$ & $5737 \mathrm{l}$ \\
28 & 12,8 & $171,16 \mathrm{c}$ & $5843 \mathrm{l}$ \\
34 & 12,4 & $167,85 \mathrm{~d}$ & $5960 \mathrm{l}$ \\
21 & 12,9 & $165,50 \mathrm{~d}$ & $6043 \mathrm{k}$ \\
20 & 12,3 & $162,50 \mathrm{e}$ & $6154 \mathrm{k}$ \\
37 & 13,1 & $162,35 \mathrm{e}$ & $6164 \mathrm{k}$ \\
24 & 13,3 & $158,71 \mathrm{f}$ & $6302 \mathrm{j}$ \\
35 & 13,2 & $158,32 \mathrm{f}$ & $6319 \mathrm{j}$ \\
36 & 13,3 & $150,68 \mathrm{~g}$ & $6638 \mathrm{i}$ \\
27 & 11,5 & $150,53 \mathrm{~g}$ & $6646 \mathrm{i}$ \\
52 & 12,7 & $147,60 \mathrm{~h}$ & $6776 \mathrm{~h}$ \\
41 & 12,6 & $146,35 \mathrm{~h}$ & $6839 \mathrm{~h}$ \\
23 & 13,1 & $144,78 \mathrm{~h}$ & $6915 \mathrm{~h}$ \\
26 & 12,9 & $144,31 \mathrm{~h}$ & $6931 \mathrm{~h}$ \\
46 & 13,0 & $135,48 \mathrm{i}$ & $7391 \mathrm{~g}$ \\
53 & 12,7 & $128,05 \mathrm{j}$ & $7812 \mathrm{f}$ \\
31 & 11,7 & $123,17 \mathrm{k}$ & $8125 \mathrm{e}$ \\
44 & 12,7 & $122,03 \mathrm{k}$ & $8195 \mathrm{e}$ \\
39 & 11,5 & $116,45 \mathrm{l}$ & $8592 \mathrm{~d}$ \\
12 & 13,3 & $115,59 \mathrm{l}$ & $8656 \mathrm{~d}$ \\
45 & 11,7 & $115,15 \mathrm{l}$ & $8690 \mathrm{~d}$ \\
10 & 13,5 & $105,75 \mathrm{~m}$ & $9456 \mathrm{c}$ \\
6 & 12,0 & $101,46 \mathrm{n}$ & $9859 \mathrm{~b}$ \\
43 & 11,6 & $100,98 \mathrm{n}$ & $9903 \mathrm{~b}$ \\
50 & 13,1 & $97,29 \mathrm{n}$ & $10293 \mathrm{a}$ \\
\hline
\end{tabular}

Médias seguidas pela mesma letra minúscula na coluna, não diferem entre si pelo teste de Scott-Knott a 5\% de probabilidade 
complementa as Regras para Análise de Sementes com as particularidades das espécies florestais (BRASIL, 2013).

Verifica-se pela análise de variância para a qualidade fisiológica das sementes provenientes das 28 matrizes de catingueira que houve efeito significativo ao nível de $5 \%$ de probabilidade pelo teste $\mathrm{F}$ para todas as variáveis estudadas (Tabela 3 ).

Os resultados relativos ao potencial fisiológico inicial das sementes oriundas de 28 matrizes de Poincianella pyramidalis estão na Tabela 4. Pelos valores obtidos no teste de germinação verifica-se que 6 matrizes $(10 ; 12 ; 22 ; 44 ; 46$ e 53) expressaram percentual de germinação superior às demais matrizes estudados. Vale ressaltar que o teste de germinação é o mais utilizado para avaliar a qualidade fisiológica das sementes de várias espécies, no entanto, ele é realizado em condições ótimas, e nem sempre reflete o comportamento destas no campo, conforme mostra os resultados de porcentagem de emergência em campo (Tabela 4), onde os maiores valores foram obtidos nas sementes oriundas das matrizes $22 ; 12 ; 46$ e 53, com as provenientes das matrizes 10 e 44 , que estavam no grupo das com maior porcentagem de germinação, sendo a porcentagem de emergência inferior.

Variabilidade na qualidade fisiológica das sementes de Tabebuia chrysotricha (Mart. Ex A. DC.) StandI. proveniente de diferentes matrizes também foram observados nos testes de germinação e de envelhecimento acelerado (SANTOS et al., 2009).

A maior expressão do vigor foi obtida pelo aumento da velocidade de formação das plântulas, observada pela maior porcentagem de plântulas na primeira contagem proveniente das matrizes 12; 22; 46; 10 e 44 e, pelo índice de velocidade de germinação com resultados semelhantes àqueles da porcentagem de germinação, incluindo as matrizes 36; 14 e 27 entre o grupo com as maiores médias. Entretanto, em sementes de Solanum sessiliflorum Dunal, em lotes com melhor desempenho na ocasião da primeira contagem de germinação os resultados do índice de velocidade de germinação foram inferiores (PEREIRA; MARTINS FILHO, 2010).

Os resultados da avaliação do desempenho das plântulas, em relação ao comprimento, podem ser observados na Tabela 4, onde se verifica que as sementes oriundas das matrizes demonstraram superioridade, exceto nas matrizes $14 ; 27 ; 28 ; 37$; 41; 43 e 50 houve redução no desempenho. Para a massa de matéria seca de plântulas, o maior valor foi observado naquela proveniente das sementes da matriz 22 (61,3 mg plântula $\left.{ }^{-1}\right)$, diferindo significativamente dos demais.

Em relação aos dados de emergência, observa-se que as matrizes 22; 12; 46 e 53 proporcionaram valores significativamente superiores àqueles obtidos nas demais matrizes, enquanto quando o vigor foi avaliado pela primeira contagem e índice de velocidade de emergência, o maior vigor foi encontrado nas sementes procedentes das matrizes 12 e 22 .

Dessa forma, das 28 matrizes de $P$. pyramidalis pesquisadas, baseado nos dados de germinação das seis melhores, foi possível separar as mesmas em diferentes níveis de vigor, sendo, pelo teste de PCG as matrizes 22; $12 ; 46 ; 10$ e 44 superiores a matriz 53; pelo teste de MS a matriz 22 superior as matrizes $12 ; 46 ; 10 ; 44$ e 53; pelo teste de $\mathrm{E}(\%)$ as matrizes $22 ; 12 ; 46$ e 53 superiores as matrizes 10 e 44; pelos testes PCE (\%) e IVE as matrizes 22 e 12 superiores as matrizes 46; 10; 44 e 53. Pelos testes de IVG e CP não foi possível observar diferença significativa quanto ao nível de vigor das matrizes (Tabela 4).

Os resultados relativos ao teor de água inicial das sementes e os teores atingidos após os períodos de envelhecimento acelerado, tradicional e em solução salina, estão apresentados na Tabela 5. No início do teste houve variação quanto ao teor de água inicial das sementes, entre 13,3 (matrizes 10 e 46) a 12,4\% (matriz 22), entretanto, essa diferença $(1,9 \%)$ não afetou significativamente os resultados obtidos nos testes fisiológicos, como recomendado por Marcos Filho (2005).

Tabela 3 - Resumo da análise de variância de sementes de vinte oito matrizes de P. pyramidalis, provenientes de Soledade - PB

\begin{tabular}{cccccccccc}
\hline \multirow{2}{*}{ FV } & \multirow{2}{*}{ GL } & \multicolumn{9}{c}{ Quadrado médio } \\
\cline { 3 - 9 } & & G & PCG & IVG & CP & MS & E & PCE & IVE \\
\hline Matrizes & 27 & $810,19^{*}$ & $709,59^{*}$ & $2,73^{*}$ & $64,7^{*}$ & $538,92^{*}$ & $535,17^{*}$ & $467,4^{*}$ & $0,78^{*}$ \\
Resíduo & 84 & 51,46 & 47,1 & 1,14 & 11,4 & 84,72 & 52,47 & 46,14 & 0,1 \\
CV $(\%)$ & & 19,5 & 20,7 & 20,3 & 27,9 & 29,4 & 21,7 & 22,1 & 34,0 \\
\hline
\end{tabular}

FV - fontes de variação; GL - graus de liberdade; G - germinação (\%); PCG - primeira contagem de germinação (\%); IVG - índice de velocidade de germinação; CP - comprimento de plântulas (cm), MS - massa de matéria seca de plântula (mg); E - emergência em campo (\%); PCE - primeira contagem de emergência (\%); IVE - índice de velocidade de emergência; *significativo a 5\% pelo teste $\mathrm{F}$ 
Tabela 4 - Germinação (G), primeira contagem de germinação (PCG), índice de velocidade de germinação (IVG), comprimento de plântulas (CP), massa seca de plântula (MS) emergência (E), primeira contagem de emergência (PCE) e índice de velocidade de emergência (IVE) de vinte oito matrizes de P. pyramidalis, provenientes de Soledade-PB

\begin{tabular}{|c|c|c|c|c|c|c|c|c|}
\hline Matrizes & $\mathrm{G}(\%)$ & PCG $(\%)$ & IVG & $\mathrm{CP}$ (cm/plântula) & MS (mg/plântula) & $\mathrm{E}(\%)$ & $\operatorname{PCE}(\%)$ & IVE \\
\hline 22 & $56 \mathrm{a}$ & $53 \mathrm{a}$ & $3,757 \mathrm{a}$ & $14,93 \mathrm{a}$ & $61,3 \mathrm{a}$ & $50 \mathrm{a}$ & $47 \mathrm{a}$ & $1,943 \mathrm{a}$ \\
\hline 12 & $56 \mathrm{a}$ & $54 \mathrm{a}$ & $3,779 \mathrm{a}$ & $13,57 \mathrm{a}$ & $38,0 \mathrm{~b}$ & $59 \mathrm{a}$ & $55 \mathrm{a}$ & $2,206 \mathrm{a}$ \\
\hline 46 & $54 \mathrm{a}$ & $50 \mathrm{a}$ & $3,465 \mathrm{a}$ & $13,42 \mathrm{a}$ & $42,3 \mathrm{~b}$ & $39 a$ & $30 \mathrm{~b}$ & $1,415 \mathrm{~b}$ \\
\hline 10 & $48 \mathrm{a}$ & $43 \mathrm{a}$ & $2,732 \mathrm{a}$ & $13,71 \mathrm{a}$ & $32,3 \mathrm{~b}$ & $31 \mathrm{~b}$ & $30 \mathrm{~b}$ & $1,245 \mathrm{~b}$ \\
\hline 44 & $47 \mathrm{a}$ & $41 \mathrm{a}$ & $2,861 \mathrm{a}$ & $14,06 \mathrm{a}$ & $36,8 \mathrm{~b}$ & $31 \mathrm{~b}$ & $31 \mathrm{~b}$ & $1,239 \mathrm{~b}$ \\
\hline 53 & $42 \mathrm{a}$ & $38 \mathrm{~b}$ & $2,684 \mathrm{a}$ & $15,49 \mathrm{a}$ & $40,3 \mathrm{~b}$ & $37 \mathrm{a}$ & $35 \mathrm{~b}$ & $1,413 b$ \\
\hline 29 & $38 \mathrm{~b}$ & $30 \mathrm{~b}$ & $2,039 \mathrm{~b}$ & $18,42 \mathrm{a}$ & $39,0 \mathrm{~b}$ & $25 \mathrm{~b}$ & $20 \mathrm{c}$ & $0,941 \mathrm{c}$ \\
\hline 36 & $38 \mathrm{~b}$ & $36 \mathrm{~b}$ & $2,387 \mathrm{a}$ & $15,06 \mathrm{a}$ & $40,0 \mathrm{~b}$ & $23 \mathrm{~b}$ & $19 \mathrm{c}$ & $0,854 \mathrm{c}$ \\
\hline 35 & $37 \mathrm{~b}$ & $22 \mathrm{c}$ & $1,594 \mathrm{~b}$ & $15,47 \mathrm{a}$ & $31,0 \mathrm{~b}$ & $16 \mathrm{~b}$ & $16 \mathrm{c}$ & $0,710 \mathrm{c}$ \\
\hline 15 & $37 \mathrm{~b}$ & $33 \mathrm{~b}$ & $2,121 \mathrm{~b}$ & $13,06 \mathrm{a}$ & $36,0 \mathrm{~b}$ & $28 \mathrm{~b}$ & $25 \mathrm{c}$ & $1,192 b$ \\
\hline 39 & $37 \mathrm{~b}$ & $28 \mathrm{~b}$ & $1,869 \mathrm{~b}$ & $15,86 \mathrm{a}$ & $33,0 \mathrm{~b}$ & $27 \mathrm{~b}$ & $20 \mathrm{c}$ & $0,913 \mathrm{c}$ \\
\hline 34 & $36 \mathrm{~b}$ & $26 \mathrm{~b}$ & $1,761 \mathrm{~b}$ & $16,17 \mathrm{a}$ & $37,0 \mathrm{~b}$ & $25 \mathrm{~b}$ & $19 \mathrm{c}$ & $0,895 \mathrm{c}$ \\
\hline 52 & $35 \mathrm{~b}$ & $30 \mathrm{~b}$ & $1,892 \mathrm{~b}$ & $14,41 \mathrm{a}$ & $15,0 \mathrm{c}$ & $19 \mathrm{~b}$ & $19 \mathrm{c}$ & $0,998 \mathrm{c}$ \\
\hline 20 & $33 \mathrm{~b}$ & $27 \mathrm{~b}$ & $1,861 \mathrm{~b}$ & $15,02 \mathrm{a}$ & $37,0 \mathrm{~b}$ & $28 \mathrm{~b}$ & $27 \mathrm{~b}$ & $1,307 \mathrm{~b}$ \\
\hline 31 & $33 \mathrm{~b}$ & $29 \mathrm{~b}$ & $1,613 \mathrm{~b}$ & $16,22 \mathrm{a}$ & $36,0 \mathrm{~b}$ & $22 \mathrm{~b}$ & $20 \mathrm{c}$ & $0,986 \mathrm{c}$ \\
\hline 6 & $30 \mathrm{~b}$ & $21 \mathrm{c}$ & $1,457 \mathrm{~b}$ & $12,79 \mathrm{a}$ & $24,0 \mathrm{c}$ & $34 \mathrm{~b}$ & $31 \mathrm{~b}$ & $1,533 b$ \\
\hline 28 & $29 \mathrm{~b}$ & $19 \mathrm{c}$ & $1,291 \mathrm{~b}$ & $6,48 \mathrm{~b}$ & $34,0 \mathrm{~b}$ & $23 \mathrm{~b}$ & $19 \mathrm{c}$ & $0,765 \mathrm{c}$ \\
\hline 26 & $28 \mathrm{~b}$ & $18 \mathrm{c}$ & $1,314 \mathrm{~b}$ & $15,40 \mathrm{a}$ & $31,0 \mathrm{~b}$ & $19 \mathrm{~b}$ & $15 \mathrm{c}$ & $0,780 \mathrm{c}$ \\
\hline 23 & $25 \mathrm{~b}$ & $20 \mathrm{c}$ & $1,251 \mathrm{~b}$ & $11,72 \mathrm{a}$ & $28,0 \mathrm{~b}$ & $27 \mathrm{~b}$ & $20 \mathrm{c}$ & $0,930 \mathrm{c}$ \\
\hline 21 & $22 \mathrm{~b}$ & $21 \mathrm{c}$ & $1,428 \mathrm{~b}$ & $12,55 \mathrm{a}$ & $37,0 \mathrm{~b}$ & $20 \mathrm{~b}$ & $19 \mathrm{c}$ & $0,955 \mathrm{c}$ \\
\hline 24 & $22 \mathrm{~b}$ & $21 \mathrm{c}$ & $1,341 \mathrm{~b}$ & $13,71 \mathrm{a}$ & $39,0 \mathrm{~b}$ & $24 \mathrm{~b}$ & $24 \mathrm{c}$ & $1,243 b$ \\
\hline 50 & $19 \mathrm{c}$ & $15 \mathrm{c}$ & $0,812 \mathrm{~b}$ & $5,83 \mathrm{~b}$ & $21,0 \mathrm{c}$ & $5 c$ & $5 \mathrm{~d}$ & $0,281 \mathrm{~d}$ \\
\hline 14 & $14 \mathrm{c}$ & $13 \mathrm{~d}$ & $2,887 \mathrm{a}$ & $6,88 \mathrm{~b}$ & $19,0 \mathrm{c}$ & $11 \mathrm{c}$ & $11 \mathrm{~d}$ & $0,565 \mathrm{~d}$ \\
\hline 45 & $13 \mathrm{c}$ & $11 \mathrm{~d}$ & $0,629 \mathrm{~b}$ & $15,51 \mathrm{a}$ & $22,0 \mathrm{c}$ & $20 \mathrm{~b}$ & $18 \mathrm{c}$ & $0,713 \mathrm{c}$ \\
\hline 27 & $11 \mathrm{c}$ & $10 \mathrm{~d}$ & $2,371 \mathrm{a}$ & $7,2 \mathrm{~b}$ & $16,0 \mathrm{c}$ & $10 \mathrm{c}$ & $9 \mathrm{~d}$ & $0,419 \mathrm{~d}$ \\
\hline 41 & $10 \mathrm{c}$ & $10 \mathrm{~d}$ & $2,112 b$ & $5,16 \mathrm{~b}$ & $17,0 \mathrm{c}$ & $26 \mathrm{~b}$ & $22 \mathrm{c}$ & $1,098 \mathrm{c}$ \\
\hline 43 & $10 \mathrm{c}$ & $9 \mathrm{~d}$ & $1,298 \mathrm{~b}$ & $5,75 \mathrm{~b}$ & $2,0 \mathrm{~d}$ & $9 c$ & $9 \mathrm{~d}$ & $0,379 \mathrm{~d}$ \\
\hline 37 & $10 \mathrm{c}$ & $8 \mathrm{~d}$ & $1,352 \mathrm{~b}$ & $4,95 \mathrm{~b}$ & $16,0 \mathrm{c}$ & $30 \mathrm{~b}$ & $30 \mathrm{~b}$ & $1,612 b$ \\
\hline
\end{tabular}

Médias seguidas pela mesma letra minúscula na coluna, não diferem entre si pelo teste de Scott-Knott a $5 \%$ de probabilidade

Um aspecto importante a ser considerado no teste de envelhecimento acelerado é a diferença na absorção de água pelas sementes, pois, quando expostas a atmosfera úmida ocorrem variações no teor de água destas (TUNES et al., 2011). Essas variações podem provocar mudanças na intensidade de deterioração, pois quanto maior o teor de água das sementes, maior a sensibilidade à alta temperatura e umidade relativa do ar que ocorrem durante o teste (LIMA et al., 2011).
Com relação aos resultados do teor de água das sementes após o envelhecimento acelerado (Tabela 5), independente do período empregado (24; 48 e 72 h) de exposição das sementes no método tradicional, houve acréscimo nos teores de água das seis matrizes, comparativamente aos teores de água iniciais das mesmas. Todavia, vale ressaltar que ao utilizar o método de envelhecimento acelerado com solução salina, nos mesmos períodos utilizados, ocorreu decréscimo dos 
Tabela 5 - Teor de água de sementes de P. pyramidalis (\%), antes (inicial) e depois do teste de envelhecimento acelerado tradicional e envelhecimento acelerado em solução salina, a $41^{\circ} \mathrm{C}$ por $24 ; 48$ e 72 horas

\begin{tabular}{|c|c|c|c|c|c|c|c|}
\hline \multirow{3}{*}{ Matrizes } & \multirow{3}{*}{ Inicial } & \multicolumn{3}{|c|}{$\begin{array}{l}\text { Envelhecimento acelerado } \\
\text { tradicional (EAT) }\end{array}$} & \multicolumn{3}{|c|}{$\begin{array}{l}\text { Envelhecimento acelerado com } \\
\text { solução salina (EAS) }\end{array}$} \\
\hline & & \multicolumn{6}{|c|}{ - } \\
\hline & & $24 \mathrm{~h}$ & $48 \mathrm{~h}$ & $72 \mathrm{~h}$ & $24 \mathrm{~h}$ & $48 \mathrm{~h}$ & $72 \mathrm{~h}$ \\
\hline 10 & 13,3 & 20,7 & 26,5 & 25,6 & 13,0 & 11,8 & 12,1 \\
\hline 12 & 12,5 & 26,0 & 24,8 & 26,3 & 11,7 & 11,2 & 11,6 \\
\hline 22 & 12,4 & 22,2 & 24,7 & 24,5 & 11,5 & 11,0 & 11,4 \\
\hline 44 & 12,6 & 22,9 & 30,1 & 25,2 & 11,6 & 11,2 & 11,2 \\
\hline 46 & 13,3 & 23,2 & 29,4 & 28,4 & 12,3 & 11,6 & 11,6 \\
\hline 53 & 12,5 & 24,6 & 25,5 & 31,3 & 11,9 & 11,6 & 12,4 \\
\hline
\end{tabular}

valores quando comparados aos teores de água iniciais das diferentes matrizes avaliadas.

$\mathrm{Na}$ Tabela 6 estão os resultados da análise de variância referentes à avaliação do envelhecimento acelerado das sementes oriundas de seis matrizes de $P$. pyramidalis. Verifica-se que houve efeito significativo a $5 \%$ de probabilidade pelo teste $\mathrm{F}$ em todos os períodos de exposição ao envelhecimento.

Os dados referentes ao teste de envelhecimento acelerado tradicional e em solução salina encontramse na Tabela 7, onde os resultados obtidos para esses testes indicaram a utilização da temperatura de $41{ }^{\circ} \mathrm{C}$ e o período de $24 \mathrm{~h}$ de exposição em envelhecimento acelerado tradicional como o procedimento mais adequado para a avaliação do potencial fisiológico das sementes oriundas das matrizes de $P$. pyramidalis, pois, os dados obtidos neste teste se relacionaram com os obtidos no teste de emergência em campo (Tabela 4). Cabe destacar que o envelhecimento acelerado tradicional no período de 24 horas otimiza a avaliação fisiológica das sementes em menor tempo, além de ser menos oneroso, por não necessitar da utilização do sal.
As sementes de várias espécies florestais podem se comportar de maneira diferente quando submetidas a períodos e temperaturas de envelhecimento. A exposição das sementes de Anadenanthera colubrina, a partir de $24 \mathrm{~h}$ às condições adversas de temperatura e umidade, promove redução na viabilidade (GARCIA; NOGUEIRA; ABREU, 2004).

Em trabalho desenvolvido com sementes de Caesalpinia echinata Lam., Lamarca; Leduc; Barbedo (2009) verificaram que a germinação e o vigor diminuíram progressivamente desde o início do período de envelhecimento acelerado até o último período (72 horas). Comportamento contraditório foi observado neste trabalho quando as sementes foram submetidas nos diferentes períodos de exposição e em ambos os métodos de envelhecimento acelerado utilizados.

O teste de envelhecimento acelerado com solução salina nos três períodos (24; 48 e 72 h) empregados de exposição nas sementes, apesar de terem ranqueado as mesmas, não é eficiente pelo fato de a extratificação das matrizes não se relacionarem com os dados obtidos na emergência em campo (Tabela 4). Outros autores

Tabela 6 - Resumo da análise de variância de sementes de seis matrizes de P. pyramidalis, provenientes de Soledade - PB

\begin{tabular}{lccccccc}
\hline \multirow{2}{*}{ FV } & \multirow{2}{*}{ GL } & \multicolumn{7}{c}{ Quadrado médio } \\
\cline { 3 - 7 } & & EAT 24h & EAT 48h & EAT 72h & EAS 24h & EAS 48h & EAS 72h \\
\hline Matrizes & 5 & $377,6^{*}$ & $455,6^{*}$ & $239,06^{*}$ & $805,5^{*}$ & $574,0^{*}$ & $680,6^{*}$ \\
Resíduo & 18 & 18,2 & 24,2 & 32,2 & 29,5 & 17,1 & 39,3 \\
CV $(\%)$ & & 11,8 & 11,5 & 13,7 & 18,6 & 16,8 & 25,9 \\
\hline
\end{tabular}

EAT - envelhecimento acelerado tradicional (24; 48 e 72 horas); EAS - envelhecimento acelerado em solução salina (24, 48 e 72 horas); *significativo a $5 \%$ pelo teste $\mathrm{F}$ 
Tabela 7 - Porcentagem de germinação de sementes de seis matrizes de P. pyramidalis, após submetidas a três períodos de exposição (24, 48 e 72 horas) no teste de envelhecimento acelerado tradicional (EAT) e envelhecimento acelerado em solução salina (EAS) à temperatura de $41^{\circ} \mathrm{C}$

\begin{tabular}{|c|c|c|c|c|c|c|}
\hline \multirow{3}{*}{ Matrizes } & \multicolumn{3}{|c|}{ Envelhecimento acelerado tradicional } & \multicolumn{3}{|c|}{ Envelhecimento acelerado com solução salina } \\
\hline & \multicolumn{6}{|c|}{ " } \\
\hline & EAT $24 \mathrm{~h}$ & EAT $48 h$ & EAT $72 \mathrm{~h}$ & EAS $24 h$ & EAS $48 \mathrm{~h}$ & EAS $72 \mathrm{~h}$ \\
\hline & \multicolumn{6}{|c|}{$\%$} \\
\hline 10 & $29 \mathrm{~b}$ & $40 \mathrm{c}$ & $30 \mathrm{a}$ & $1 \mathrm{~b}$ & $15 \mathrm{~b}$ & $9 \mathrm{~b}$ \\
\hline 12 & $51 \mathrm{a}$ & $48 \mathrm{~b}$ & $53 \mathrm{a}$ & 36 a & $20 \mathrm{~b}$ & $28 \mathrm{a}$ \\
\hline 22 & $40 \mathrm{a}$ & $59 \mathrm{a}$ & $46 \mathrm{a}$ & $37 \mathrm{a}$ & $48 \mathrm{a}$ & $43 \mathrm{a}$ \\
\hline 44 & $23 \mathrm{~b}$ & $34 \mathrm{~d}$ & $39 a$ & $30 \mathrm{a}$ & $18 \mathrm{~b}$ & $14 \mathrm{~b}$ \\
\hline 46 & $39 a$ & $45 \mathrm{~b}$ & $39 a$ & $39 a$ & $21 \mathrm{~b}$ & $17 \mathrm{~b}$ \\
\hline 53 & $44 \mathrm{a}$ & $29 \mathrm{~d}$ & $40 \mathrm{a}$ & $32 \mathrm{a}$ & $25 \mathrm{~b}$ & $34 \mathrm{a}$ \\
\hline
\end{tabular}

Médias seguidas pela mesma letra minúscula na coluna, não diferem entre si pelo teste de Scott Knott a 5\% de probabilidade

constataram ser este o método mais promissor para classificar lotes de sementes de amendoim (ROSSETO; LIMA; GUIMARAES, 2004), grama bermuda (SILVA et al., 2010) e trigo (PEDROSO et al., 2010) em diferentes níveis de vigor.

\section{CONCLUSÕES}

1. A qualidade fisiológica das sementes de Poincianella pyramidalis varia entre as matrizes de uma única área de coleta;

2. O teste de envelhecimento acelerado, utilizado no método tradicional, no período de 24 horas e à temperatura de $41{ }^{\circ} \mathrm{C}$ mostra ser o teste de vigor mais eficiente na avaliação do potencial fisiológico das sementes de $P$. pyramidalis.

\section{AGRADECIMENTOS}

À CAPES (Coordenação de Aperfeiçoamento de Pessoal de Nível Superior) e ao CNPq (Conselho Nacional de Desenvolvimento CientíficoeTecnológico), pelas bolsas concedidas para pesquisadores e estudantes de Pós-Graduação.

\section{REFERÊNCIAS}

BRASIL. Ministério da Agricultura, Pecuária e Abastecimento. Regras para análise de sementes. Secretaria de Defesa Agropecuária. Brasília: MAPA/ACS5 2009. 395 p.
BRASIL, Ministério da Agricultura, Pecuária e Abastecimento. Instruções para análise de sementes de espécies florestais, de 17 de janeiro de 2013, Brasília: MAPA, 2013. 98 p.

DAVIDE, A. C.; SILVA, E. A. A. Produção de sementes e mudas de espécies florestais. Lavras: Ed. UFLA, 2008. 174 p.

DUTRA, A. S.; VIEIRA, R. D. Envelhecimento acelerado como teste de vigor para sementes de milho e soja. Ciência Rural, v. 34, n. 3, p. 715-721, 2004.

GARCiA, L. C.; NOGUEIRA, A. C.; ABreU, D. C. A. Influência do envelhecimento acelerado no vigor de sementes de Anadenanthera colubrina (Vellozo) Brenan - Mimosaceae. Ciência Florestal, v. 14, n. 1, p. 85-90, 2004.

GUEDES, R. S. et al. Resposta fisiológica de sementes de Erythrina velutina Willd. ao envelhecimento acelerado. Semina: Ciências Agrárias, v. 30, n. 2, p. 323-330, 2009.

HIGA, A. R.; SILVA, L. D. Pomar de sementes de espécies florestais nativas. Curitiba, PR - FUPEF. 2006.

JIANHUA, Z.; McDONALD, M. D. The saturated salt accelerated aging test for small-seeded crops. Seed Science and Technology, v. 25, n. 1, p. 123-131, 1996.

LAMARCA, E. V.; LEDUC, S. N. M.; BARBEDO, C. J. Viabilidade e vigor de sementes de Caesalpinia echinata Lam. (pau-brasil - Leguminosae) pelo teste de tetrazólio. Revista Brasileira de Botânica, v. 32, n. 4, p. 793-803, 2009.

LIMA, C. B. et al. Germinação e envelhecimento acelerado na análise da qualidade fisiológica de sementes de alfavacacravo. Semina: Ciências Agrárias, v. 32, n. 3, p. 865-874, 2011.

LIMA, C. R. et al. Temperaturas e substratos na germinação de sementes de Caesalpinia pyramidalis Tul. Revista Brasileira de Sementes, v. 33, n. 2, p. 216-222, 2011. 
MAGUIRE, J. D. Speed of germination: aid in selection and evaluation for seedling emergence and vigour. Crop Science, v. 2, n. 2, p. 176-177, 1962.

MAIA, G. N. Catingueira. In: MAIA, G. N. Caatinga: árvores e arbustos e suas utilidades. São Paulo: Leitura e Arte, 2004. p. 159-169.

MARCOS FILHO, J. Testes de vigor: importância e utilização. In: KRZYZANOWSKI, F.C.; VIEIRA, R. D.; FRANÇA-NETO, J. B. (eds.). Vigor de sementes: conceitos e testes. Londrina: ABRATES, 1999. p. 1.1-1.21.

MARCOS FILHO, J. Fisiologia de sementes de plantas cultivadas. Piracicaba: FEALQ, 2005. 495p.

NAKAGAWA, J. Testes de vigor baseados na avaliação das plântulas. In: VIEIRA, R. D.; CARVALHO, N. M. Testes de Vigor em Sementes. Jaboticabal: FUNEP, 1999. p. 49-85.

OLIVEIRA, O. S. Tecnologia de sementes florestais. Curitiba: Imprensa Universitária, 2007. 185p.

PASSOS, M. A. A. et al. Luz, substrato e temperatura na germinação de sementes de cedro-vermelho. Pesquisa Agropecuária Brasileira, v. 43, n. 2, p. 281-284, 2008.

PEDROSO, D. C. et al. Envelhecimento acelerado em sementes de trigo. Ciência Rural, v. 40, n. 11, p. 2389-2392, 2010.
PEREIRA, M. D.; MARTINS FILHO, S. Envelhecimento acelerado em sementes de cubiu (Solanum sessiliflorum Dunal). Pesquisa Agropecuária Tropical, v. 40, n. 3, p. 251256, 2010.

ROSSETO, C. A. V.; LIMA, T. M.; GUIMARAES, E. C. Envelhecimento acelerado e deterioração controlada em sementes de amendoim. Pesquisa Agropecuária Brasileira, v. 39, n. 8 , p. $795-801,2004$.

SANTOS, F. S. et al. Biometria e qualidade fisiológica de sementes de diferentes matrizes de Tabebuia chrysotricha (Mart. Ex A. DC.) Standl. Scientia Forestalis, v. 37, n. 82, p. 163-173, 2009.

SILVA, C. B. et al. Teste de envelhecimento acelerado para avaliação do potencial fisiológico em sementes de grama bermuda. Revista Brasileira de Sementes, v. 32, n. 2, p. 102107, 2010.

TORRES, S. B.; NEGREIROS, M. Z. Envelhecimento acelerado em sementes de berinjela. Revista Brasileira de Sementes, v. 30, n. 2, p. 209-213, 2008.

TUNES, L. M. et al. Envelhecimento acelerado em sementes de azevém com e sem solução salina e saturada. Ciência Rural, v. 41, n. 1, p. 33-37, 2011. 